\title{
Medium-induced color flow softens hadronization
}

\author{
Andrea Beraudo, ${ }^{1,2}$ José Guilherme Milhano, ${ }^{1,3}$ and Urs Achim Wiedemann ${ }^{1}$ \\ ${ }^{1}$ Physics Department, Theory Unit, CERN, CH-1211 Genève 23, Switzerland \\ ${ }^{2}$ Centro Studi e Ricerche "Enrico Fermi," Piazza del Viminale 1, I-00184 Roma, Italy \\ ${ }^{3}$ CENTRA, Instituto Superior Técnico, Universidade Técnica de Lisboa, Av. Rovisco Pais, P-1049-001 Lisboa, Portugal
}

(Received 23 September 2011; published 5 March 2012)

\begin{abstract}
Medium-induced parton energy loss, resulting from gluon exchanges between the QCD matter and partonic projectiles, is expected to underlie the strong suppression of jets and high- $p_{T}$ hadron spectra observed in ultrarelativistic heavy-ion collisions. Here, we present the first color-differential calculation of parton energy loss. We find that color exchange between medium and projectile decoheres the color of radiated gluons from that of the most energetic fragments and enhances parametrically the invariant mass of energetic color singlet clusters in the parton shower. These effects are seen in more than half of the medium-modified parton branchings. As shown for a cluster hadronization model and as argued for a Lund string fragmentation model, this leads to additional softening of hadronic spectra. Compared to standard parton energy loss calculations, a lower density of the QCD matter is then needed to account for the nuclear modification factor at the Large Hadron Collider.
\end{abstract}

DOI: 10.1103/PhysRevC.85.031901

PACS number(s): 12.38.Mh, 13.87.Fh, 25.75.Bh, 21.65.Qr

High-transverse-momentum partons $\left(p_{T} \gg 10 \mathrm{GeV}\right)$ produced in heavy-ion collisions interact with the QCD matter in the collision region while branching. This interaction is thought to cause the strong medium modification of hadronic spectra and jets measured in heavy-ion collisions at the Large Hadron Collider (LHC) and at the Relativistic Heavy Ion Collider. The modeling of this jet quenching phenomenon has focused so far on medium-induced parton energy loss prior to hadronization [1], assuming that, for sufficiently high $p_{T}$, hadronization occurs time delayed and thus outside the medium. However, if the color flow of a parton shower is modified within the medium, then hadronization can be affected irrespective of when it occurs. Here, we analyze the medium-induced color flow in a standard parton-energy-loss calculation. Compared to the current modeling of parton energy loss [2-7], this will be seen to result in a characteristic softening of the ensuing hadronization. It may, thus, affect significantly the extraction of medium properties from the measured nuclear modification factor at the LHC [8-10].

We start by considering an elementary building block of a parton shower, the $q \rightarrow q g$ parton splitting. For a small light-cone energy $k^{+}$of the gluon compared to the parent parton, $x \equiv k^{+} / p^{+} \ll 1$, and for transverse gluon momentum $\mathbf{k}$ with $\mathbf{K}_{\mathbf{0}} \equiv \mathbf{k} / \mathbf{k}^{2}$, the gluon spectrum reads, to leading order in $\alpha_{s}$,

$$
k^{+} \frac{d I^{\mathrm{vac}}}{d k^{+} d \mathbf{k}}=\frac{\alpha_{s} C_{R}}{\pi^{2}} \mathbf{K}_{\mathbf{0}}^{2} .
$$

In the presence of QCD matter, interactions between projectile parton and medium result in a modified spectrum $d I^{\mathrm{rad}} \equiv$ $d I^{\mathrm{vac}}+d I^{\mathrm{med}}$ that can be written as an expansion in powers of the ratio $\zeta \equiv L^{+} / \lambda_{\text {el }}^{+}$of the in-medium path length $L^{+}$and an elastic mean free path $\lambda_{\text {el }}^{+}$(for details, see Ref. [1]). To first order in this opacity expansion, the medium-induced radiation spectrum reads

$$
k^{+} \frac{d I^{\text {med }}}{d k^{+} d \mathbf{k}}=\zeta \frac{\alpha_{s} C_{R}}{\pi^{2}}\left\langle\left(\left(\mathbf{K}_{\mathbf{0}}-\mathbf{K}_{\mathbf{1}}\right)^{2}-\mathbf{K}_{\mathbf{0}}^{2}+\mathbf{K}_{\mathbf{1}}^{2}\right) \mathcal{T}_{I}\right\rangle
$$

where $\langle\cdots\rangle$ denotes averaging over the transverse-momentum transfer $\mathbf{q}$ of a single interaction and $\mathbf{K}_{\mathbf{1}} \equiv(\mathbf{k}-\mathbf{q}) /(\mathbf{k}-$ $\mathbf{q})^{2}$. Medium-induced interference effects enter via the factor

$$
\mathcal{T}_{I}=\left[1-\frac{\sin \left(\omega_{1}^{-} L^{+}\right)}{\omega_{1}^{-} L^{+}}\right]=\left\{\begin{array}{lll}
1 & \text { for } & 1 / \omega_{1}^{-} \ll L^{+} \\
0 & \text { for } & 1 / \omega_{1}^{-} \gg L^{+}
\end{array} .\right.
$$

Thus, the medium-modification $d I^{\text {med }}$ can occur only for quanta of sufficiently small formation time $1 / \omega_{1}^{-} \equiv 2 k^{+} /(\mathbf{k}-$ $\mathbf{q})^{2} \ll L^{+}$. To first order in opacity and at large $N_{c}$, we identify three contributions with distinct color flow,

$$
d I^{\mathrm{med}}=d I_{\mathrm{virt}}^{\mathrm{med}}+d I_{\mathrm{low} M}^{\mathrm{med}}+d I_{\mathrm{high} M}^{\mathrm{med}},
$$

where $d I_{\text {virt }}^{\text {med }}$ arises from probability-conserving virtual corrections that change neither color flow nor kinematic distributions in the projectile. The contributions $d I_{\mathrm{low} M}^{\mathrm{med}}$ and $d I_{\mathrm{high} M}^{\mathrm{med}}$ are characterized as follows. For a projectile with (light-cone) energy much larger than that of its target scattering partner, $p^{+} \gg t^{+}$, and for $x \ll 1$, the most energetic parton in the final state in Fig. 1 is defined unambiguously and carries energy $p_{f}^{+} \equiv(1-x) p^{+}$. Distributing the energy of the gluon equally between its $q$ and $\bar{q}$ legs in the large $N_{c}$ limit (our final conclusions will not depend on this assumption), the energy and invariant mass of the most energetic singlet cluster in a vacuum splitting read

$$
P_{\mathcal{C}_{\text {vac }}}^{+} \simeq(1+x / 2) p_{f}^{+}, \quad M_{\mathcal{C}_{\text {vac }}}^{2} \simeq \mathbf{k}^{2} / 2 x
$$

These relations hold for the vacuum contribution of Eq. (1), the virtual correction $d I_{\text {virt }}^{\text {med }}$ in Eq. (4), and for $d I_{\text {low } M}^{\text {med }}$ (in the latter case, the distributions in $x$ and $\mathbf{k}$ contain information about the medium modification).

In contrast, for $d I_{\mathrm{high} M}^{\mathrm{med}}$ color flows from the most energetic final-state parton directly to a target component of momentum $t$, the gluon is decohered in color and

$$
P_{\mathcal{C}_{\text {targ }}}^{+} \simeq p_{f}^{+}, \quad M_{\mathcal{C}_{\mathrm{targ}}}^{2}=\left(p_{f}+t\right)^{2} \simeq p^{+} Q_{T},
$$




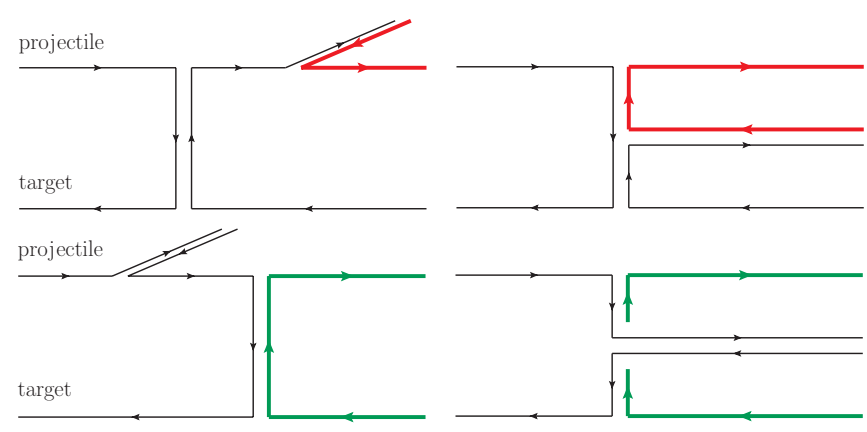

FIG. 1. (Color online) $N=1$ opacity diagrams for gluon radiation from a projectile quark in the large- $N_{c}$ limit. The most energetic color-singlet clusters are denoted by thicker lines and correspond to color flow between projectile components (upper diagrams, contribution $d I_{\text {low } M}^{\text {med }}$ ) or between projectile and target (lower diagrams, contribution $d I_{\text {high } M}^{\mathrm{med}}$ ). Diagrams on the right-hand side include a three-gluon vertex.

where $Q_{T} \equiv \sqrt{2} E_{\mathrm{th}}$ and $E_{\mathrm{th}}$ is the typical (thermal) energy of the target component. The invariant mass in Eq. (6) is parametrically larger than in the vacuum case [Eq. (5)] by a factor $\sqrt{p^{+}}$. This motivates the choice of subscripts in $d I_{\text {low } M}^{\text {med }}$ and $d I_{\text {high } M}^{\text {med }}$.

One can determine the kinematic conditions and time scales required for $d I_{\mathrm{high} M}^{\mathrm{med}}$ to contribute. The color-inclusive sum [Eq. (4)] depends on the formation time $1 / \omega_{1}^{-}$. To first order in opacity, we find that the color-differential contributions on the right-hand side of Eq. (4) carry interference factors involving two formation times $1 / \omega_{1}^{-}$and $1 / \omega_{0}^{-} \equiv 2 k^{+} / \mathbf{k}^{2}$ (see Ref. [11] for the full result). Here, we focus on the relevant limiting cases.

If parton energy loss becomes negligible $\left(\omega_{1}^{-} L^{+} \ll 1\right)$ and if the formation time of the final-state gluon is large $\left(\omega_{0}^{-} L^{+} \ll\right.$ 1), then

$$
\begin{aligned}
& k^{+} \frac{d I_{\text {virt }}^{\text {med }}}{d k^{+} d \mathbf{k}}=-\zeta \frac{\alpha_{s} C_{R}}{2 \pi^{2}}\left\langle\mathbf{K}_{\mathbf{0}}{ }^{2}\right\rangle, \\
& k^{+} \frac{d I_{\text {low } M}^{\text {med }}}{d k^{+} d \mathbf{k}}=\zeta \frac{\alpha_{s} C_{R}}{2 \pi^{2}}\left\langle\mathbf{K}_{\mathbf{0}}{ }^{2}\right\rangle, \quad k^{+} \frac{d I_{\text {high } M}^{\text {med }}}{d k^{+} d \mathbf{k}}=0 .
\end{aligned}
$$

In this case, the color exchange between the medium and the projectile occurs predominantly at early times before the splitting and, hence, the leading fragments have vacuum-like color correlations, $d I_{\text {high } M}^{\text {med }}=0$. In contrast, in the limit $1 / \omega_{0}^{-} \ll$ $L^{+} \ll 1 / \omega_{1}^{-}$, one finds that $d I_{\text {virt }}^{\text {med }} \sim-3\left\langle\mathbf{K}_{\mathbf{0}}{ }^{2}\right\rangle, d I_{\text {low } M}^{\text {med }} \sim$ $\left\langle\mathbf{K}_{\mathbf{0}}{ }^{2}\right\rangle$, and $d I_{\text {high } M}^{\mathrm{med}} \sim 2\left\langle\mathbf{K}_{\mathbf{0}}{ }^{2}\right\rangle$. Thus, even if the color-inclusive sum [Eq. (4)] vanishes, $d I_{\mathrm{high} M}^{\mathrm{med}}$ can be significant.

If there is parton energy loss, one finds in the limit $1 / \omega_{1}^{-}, 1 / \omega_{0}^{-} \ll L^{+}$

$$
\begin{aligned}
k^{+} \frac{d I_{\mathrm{virt}}^{\mathrm{med}}}{d k^{+} d \mathbf{k}} & =-3 \zeta \frac{\alpha_{s} C_{R}}{2 \pi^{2}}\left\langle\mathbf{K}_{\mathbf{0}}^{2}\right\rangle \\
k^{+} \frac{d I_{\mathrm{low} M}^{\text {med }}}{d k^{+} d \mathbf{k}} & =\zeta \frac{\alpha_{s} C_{R}}{2 \pi^{2}}\left\langle\left(\mathbf{K}_{\mathbf{0}}-\mathbf{K}_{\mathbf{1}}\right)^{2}+\mathbf{K}_{\mathbf{1}}^{2}\right\rangle \\
k^{+} \frac{d I_{\mathrm{high} M}^{\text {med }}}{d k^{+} d \mathbf{k}} & =\zeta \frac{\alpha_{s} C_{R}}{2 \pi^{2}}\left\langle\left(\mathbf{K}_{\mathbf{0}}-\mathbf{K}_{\mathbf{1}}\right)^{2}+\mathbf{K}_{\mathbf{1}}^{2}+\mathbf{K}_{\mathbf{0}}^{2}\right\rangle .
\end{aligned}
$$

In summary, whenever the color-averaged contribution [Eq. (4)] to radiative energy loss is significant, mediuminduced color flow changes color correlations among leading partons and the invariant mass of leading singlets in more than $50 \%$ of all cases, $d I_{\text {high } M}^{\mathrm{med}}>0.5 d I^{\mathrm{med}}$.

For a gluonic projectile, one finds [11] to first order in opacity and in the large $N_{c}$ limit a similar fraction of clusters $\mathcal{C}_{\text {targ }}$ of parametrically large invariant mass [Eq. (6)]. To higher orders in opacity, multiple interactions between projectile and target enhance the fraction of color singlet clusters $\mathcal{C}_{\text {targ }}$ [11]. Therefore, the calculation above illustrates generic features of medium-modified color flow in standard models of parton energy loss.

Since color is conserved in QCD and since hadrons are color singlets, a dynamically consistent hadronization prescription must relate hadrons to color singlet fragments of the parton shower. We, therefore, expect on general kinematic grounds that the medium-modified distribution [Eq. (8)] of leading color singlets has consequences for the dynamics of hadronization. We now illustrate this point in a cluster hadronization model that encodes essential features of the prescription implemented in the MC event generator HERWIG [12]; the parton shower is evolved perturbatively to a hadronic scale ( $Q_{0} \simeq 600 \mathrm{MeV}$ in Ref. [12]) at which the entire shower is decomposed into its color singlet components ("clusters"). Clusters $\mathcal{C}$ of low invariant mass $\left(\mathcal{M}_{\mathcal{C}}<\mathcal{M}_{\text {cr }}=4 \mathrm{GeV}\right)$ are then decayed directly into pairs of hadrons, while clusters of larger invariant mass are decayed, first, into pairs of daughter clusters, $\mathcal{C} \rightarrow X Y$, until the invariant mass of the daughters satisfies the condition for decay into hadrons. In parton showers evolved in the vacuum, far more than $90 \%$ of all clusters are found to have a small invariant mass, $\mathcal{M}_{\mathcal{C}}<4 \mathrm{GeV}$ [12]. We, therefore, take in the following $\mathcal{M}_{\mathcal{C}_{\text {vac }}}<4 \mathrm{GeV}$. We want to adapt this cluster hadronization model to a mediummodified parton shower, for which at the final scale $Q_{0}$ the most energetic parton carries energy $p_{f}^{+}$. In more than $50 \%$ of these showers, color will flow from this parton directly to the target, and one finds for the most energetic cluster $\mathcal{C}_{\text {targ }}$ an invariant mass $\mathcal{M}_{\mathcal{C}_{\text {targ }}}>4 \mathrm{GeV}$ within the range $p_{f}^{+}>p_{\mathrm{cr}}^{+}=\mathcal{M}_{\mathrm{cr}}^{2} / Q_{T}$. Estimating the thermal energies of target scatterers by their ideal gas value $E_{\text {th }} \simeq 2.7 T$, we find for $T=200(500) \mathrm{MeV}$ a value of $Q_{T} \simeq 760$ (1900) $\mathrm{MeV}$ corresponding to $p_{\mathrm{cr}}^{+}=$ 21.0(8.4) GeV. Therefore, for a wide, phenomenologically relevant range of parton energies $p_{f}^{+}>p_{\mathrm{cr}}^{+}$, hadronization of $\mathcal{C}_{\text {targ }}$ involves an additional step, $\mathcal{C}_{\text {targ }} \rightarrow X Y$, that is absent in the fragmentation of cluster $\mathcal{C}_{\text {vac }}$ and that leads to a most energetic daughter cluster $X$ of energy [12]

$$
P_{X}^{+}=\left(1-\frac{Q_{0}}{\mathcal{M}_{\mathcal{C}_{\text {targ }}}}\right) p_{f}^{+}+\frac{Q_{0}}{\mathcal{M}_{\mathcal{C}_{\text {targ }}}} t^{+} .
$$

Therefore, among the distribution of final clusters $\mathcal{C}_{f}$, defined as those that decay directly into hadrons, the daughters of $\mathcal{C}_{\text {targ }}$ are significantly softer $\left(\mathcal{C}_{f}=X, P_{X}^{+}<p_{f}^{+}\right)$than $\mathcal{C}_{\text {vac }}\left(\mathcal{C}_{f}=\right.$ $\mathcal{C}_{\text {vac }}, P_{\mathcal{C}_{\text {vac }}^{+}}^{+} \geqslant p_{f}^{+}$); see Eq. (5).

To illustrate how the softening of the cluster distribution due to color flow may affect transverse-momentum spectra, we consider a sample of hadronic collisions with final-state parton showers evolved to scale $Q_{0}$. We parametrize the 
resulting transverse partonic spectrum by a power law (within the geometry of a heavy-ion collision, the large momentum component of a hard process points transverse to the beam direction),

$$
\frac{d N}{d p_{f}^{+}}=\frac{c}{p_{f}^{+n}} .
$$

A fraction $f_{t}$ of the partons in Eq. (10) are end points of clusters $\mathcal{C}_{\text {targ }}$ with color flowing directly to the target. The distribution of final clusters $\mathcal{C}_{f}$ resulting from $\mathcal{C}_{\text {targ }}$ reads,

$$
\begin{aligned}
\frac{d N^{\mathcal{C}_{f}}}{d P^{+}}= & f_{t} \int_{0}^{P_{\mathrm{cr}}^{+}} d p_{f}^{+} \delta\left(P^{+}-p_{f}^{+}\right) \frac{d N}{d p_{f}^{+}} \\
& +f_{t} \int_{P_{\mathrm{cr}}^{+}}^{\infty} d p_{f}^{+} \delta\left[P^{+}-p_{f}^{+}\left(1-\frac{Q_{0}}{\sqrt{p_{f}^{+} Q_{T}}}\right)\right] \frac{d N}{d p_{f}^{+}} \\
= & f_{t} \frac{c}{P^{+n}} F\left(P^{+}\right) \\
F\left(P^{+}\right)= & \Theta\left(P_{\mathrm{cr}}^{+}-P^{+}\right) \\
& +\Theta\left[P^{+}-\left(P_{\mathrm{cr}}^{+}-\Delta\right)\right] \frac{2\left(1+\frac{Q_{0}}{\sqrt{P^{+} Q_{T}}}\right)}{\left(2+\frac{Q_{0}}{\sqrt{P^{+} Q_{T}}}\right)}
\end{aligned}
$$

where $\Delta \equiv Q_{0} \sqrt{P_{\mathrm{cr}}^{+} / Q_{T}}$, and the last factor in Eq. (12) comes from transforming Eq. (10) from $p_{f}^{+}$to $P_{X}^{+}$with the help of Eq. (9). The first term in Eq. (12) comes from clusters $\mathcal{C}_{\text {targ }}$ of small energy $P^{+}<P_{\text {cr }}^{+}$and small invariant mass that decay directly into hadrons, $\mathcal{C}_{f}=\mathcal{C}_{\text {targ }}$. The second term comes from clusters $\mathcal{C}_{\text {targ }}$ of large invariant mass that undergo a cluster decay, $\mathcal{C}_{f}=X$. Both mechanisms contribute in an intermediate range $P_{\mathrm{cr}}^{+}-\Delta<P^{+}<P_{\mathrm{cr}}^{+}$, where one finds an enhancement $F\left(P^{+}\right)>1$. A more realistic treatment, including corrections to the limit $p_{f}^{+} \gg t^{+}$, may be expected to smooth the $\Theta$ functions in Eq. (12). Here, we steer clear of these model-dependent uncertainties by focusing on the high-energy region $P^{+}>P_{\mathrm{cr}}^{+}$where an additional cluster decay results in a suppression, $F\left(P^{+}\right)<1$. For phenomenologically motivated parameter choices, namely a power-law spectrum [Eq. (10)] with $n=6$, values $Q_{0}=600 \mathrm{MeV}$ and $\mathcal{M}_{\text {cr }}=4 \mathrm{GeV}$, typical of hadronization models, and a range of expected thermal energies, the color-induced suppression persists up to cluster energies well above $100 \mathrm{GeV}$; see Fig. 2.

Medium-effects in single inclusive hadron spectra are typically expressed in terms of the nuclear modification factor $R_{\mathrm{AA}}\left(p_{T}\right) \equiv\left(d N^{\mathrm{AA}} / d p_{T}\right) /\left(n_{\text {coll }} d N^{\mathrm{pp}} / d p_{T}\right)$. Let us denote by $R_{\mathrm{AA}}^{\mathrm{fact}}$ the nuclear modification factor calculated in a standard implementation of parton energy loss in which the quenching dynamics is factorized from hadronization and the latter is treated as in the vacuum [1]. In this case, the energy degradation due to Eq. (4) is accounted for in $R_{\mathrm{AA}}^{\text {fact }}$, whereas effects of medium-induced color flow are not. The latter effects may be included by subjecting a fraction $f_{t}$ of the hadronic yield to a further suppression [Eq. (12)] at the cluster level. We do this by relating the energy of the final clusters $\mathcal{C}_{f}$ to the hadronic transverse momentum, $P^{+}=\sqrt{2} \frac{p_{T}}{z}$, and approximating the momentum fraction $z$ carried by the hadron by the average of a boosted, isotropic two-body decay,

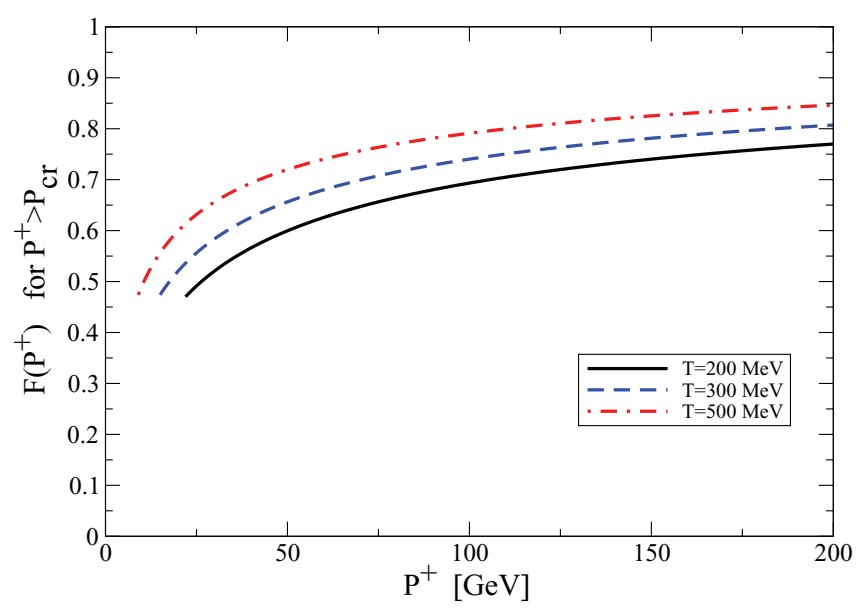

FIG. 2. (Color online) The color-induced suppression factor $F\left(P^{+}\right)$of Eq. (12) plotted for sufficiently large cluster energies $P^{+}>P_{\text {cr }}$ where additional color-flow-induced cluster decays occur.

$$
\begin{aligned}
& \langle z\rangle \sim 3 / 4, \\
& R_{\mathrm{AA}}\left(p_{T}\right) \simeq\left(1-f_{t}\right) R_{\mathrm{AA}}^{\mathrm{fact}}\left(p_{T}\right)+f_{t} F\left(\sqrt{2} \frac{4}{3} p_{T}\right) R_{\mathrm{AA}}^{\mathrm{fact}}\left(p_{T}\right) .
\end{aligned}
$$

The resulting $R_{\mathrm{AA}}$ is plotted in Fig. 3 starting from a standard implementation of parton energy loss for central $\mathrm{Pb}-\mathrm{Pb}$ collisions at the $\mathrm{LHC}$ (curve for $R_{\mathrm{AA}}^{\text {fact }}$ taken from Fig. 5 of Ref. [13]). Supplementing this model with a color-flow-induced suppression $F$ in $f_{t}=50 \%$ (100\%) of all fragmentation patterns according to Eq. (13), we find that $R_{\mathrm{AA}}$ is reduced significantly by $\sim 0.1(\sim 0.2)$ compared to $R_{\mathrm{AA}}^{\mathrm{fact}}$. A fair agreement with preliminary results of ALICE and CMS at the LHC is then found for the extreme choice $f_{t}=100 \%$. We caution, however, that in a complete phenomenological model, the value of $f_{t}$ would be determined dynamically and that the curve representing $R_{\mathrm{AA}}^{\text {fact }}$ in Fig. 3 involves

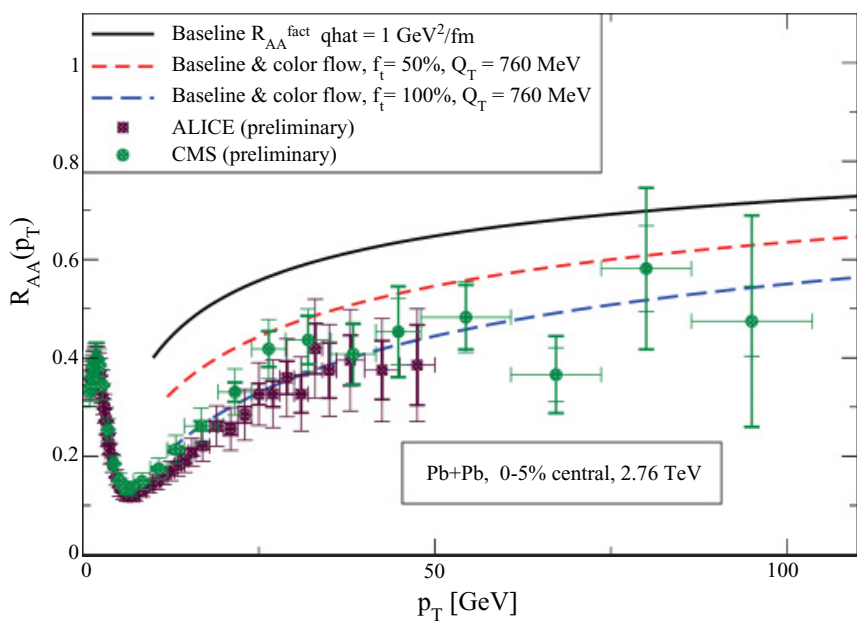

FIG. 3. (Color online) The nuclear modification factor $R_{\mathrm{AA}}\left(p_{T}\right)$. The baseline calculation of kinematic effects (solid black curve) is supplemented with the effect of color-flow modified hadronization according to Eq. (13). 
simplifications and approximations. ${ }^{1}$ Therefore, we mainly conclude that taking medium-induced color flow into account, a significant additional suppression of $R_{\mathrm{AA}}$ can be obtained without increasing the medium density.

Recent measurements of the nuclear modification factor $R_{\mathrm{AA}}$ in central $\mathrm{Pb}-\mathrm{Pb}$ collisions at the LHC have established unambiguously the rise of $R_{\mathrm{AA}}\left(p_{T}\right)$ at intermediate and high transverse momentum $p_{T} \in[10 ; 100] \mathrm{GeV}$. This has been used in several recent studies [2-7] to constrain medium properties entering the modeling of parton energy loss. It is a common feature of all these models to assume gluon exchanges between the projectile parton and the medium, while neglecting in their dynamical implementation the ensuing changes in the color structure of the parton shower. Although heuristic models of medium-modified hadronization have been discussed previously [14,15], we have assessed here the color-differential information that is contained in a QCD-based formulation of parton energy loss. From Figs. 2 and 3 we learn that mediummodified color flow may affect single inclusive hadron spectra significantly up to the highest transverse momenta $\left(p_{T}>100 \mathrm{GeV}\right.$, say). Also, remarkably, in contrast with the temperature dependence of the baseline $R_{\mathrm{AA}}^{\mathrm{fact}}$, the smaller the thermal energy in Eq. (12), the larger the color-flow-induced contribution to the suppression of $R_{\mathrm{AA}}$. As a consequence, a significantly smaller density of the medium may then be sufficient to account for the observed suppression of $R_{\mathrm{AA}}$.

${ }^{1}$ In Ref. [13], results for $R_{\mathrm{AA}}^{\mathrm{fact}}$ were calculated for $\sqrt{s_{N N}}=5.5$ $\mathrm{TeV}$. This baseline should be improved for a fully quantitative model study but is sufficient for the qualitative argument presented here, since the results for $\sqrt{s_{N N}}=5.5$ and $\sqrt{s_{N N}}=2.76 \mathrm{TeV}$ differ by much less than the curves shown for different color-flow-induced suppression in Fig. 3.
The effect of medium-modified color flow on $R_{\mathrm{AA}}$ may depend sensitively on the microscopic implementation of parton energy loss, including, e.g., the spatiotemporal embedding of the parton shower in the QCD matter and the probability that the produced partons escape the medium without gluon exchange. Figure 3 shows that this effect is potentially large and thus should be included in full microscopic simulations of parton energy loss.

Our analysis of color flow up to Eq. (8) provides novel, perturbative, and model-independent results. However, our discussion of their phenomenological implications was based on a cluster hadronization model so far. We, finally, note that qualitatively similar conclusions can be drawn from string hadronization models. In these, final-state quarks are grouped along the perturbatively specified color flow with final-state antiquarks into strings by representing all color-connected gluons as kinks along the color flow. For the contributions $d I_{\mathrm{vac}}^{\mathrm{med}}$, $d I_{\text {virt }}^{\text {med }}$, and $d I_{\text {low } M}^{\text {med }}$, the relevant Lund string represents a $q g \bar{q}$ configuration with total + energy $p_{f}^{+}$. In contrast, the leading hadronic fragments of the contribution $d I_{\text {high } M}^{\mathrm{med}}$ will come from a $q \bar{q}$ configuration with total energy $(1-x) p_{f}^{+}$, since the radiated gluon is decohered. In numerical studies within the Lund hadronization model, we have established [11] that the most energetic hadronic fragments of this latter configuration are significantly softer, even if $p_{f}^{+} \simeq 100 \mathrm{GeV}$. This is so, since the radiated gluon is decohered from this configuration while it contributes to the + component of the leading hadron in $q g \bar{q}$ strings. This indicates that the softer hadronization of the partonic contribution $d I_{\mathrm{high} M}^{\mathrm{med}}$ is a generic features that does not depend on details of the hadronization model.

We thank G. Corcella, P. Skands, and K. Zapp for helpful discussions.
[1] N. Armesto et al. (TECHQM Collaboration), arXiv:1106.1106 [hep-ph], and references therein.

[2] X.-F. Chen, T. Hirano, E. Wang, X.-N. Wang, and H. Zhang, Phys. Rev. C 84, 034902 (2011).

[3] F. Arleo, J. Phys. G 38, 124017 (2011).

[4] W. A. Horowitz and M. Gyulassy, Nucl. Phys. A 872, 265 (2011).

[5] I. P. Lokhtin, A. V. Belyaev, and A. M. Snigirev, Eur. Phys. J. C 71, 1650 (2011).

[6] B. G. Zakharov, arXiv:1105.0191 [hep-ph].

[7] O. Fochler, J. Uphoff, Z. Xu, and C. Greiner, J. Phys. G 38, 124152 (2011).

[8] H. Appelshauser, J. Phys. G 38, 124014 (2011).
[9] Y.-J. Lee, J. Phys. G 38, 124015 (2011).

[10] K. Aamodt et al. (ALICE Collaboration), Phys. Lett. B 696, 30 (2011).

[11] A. Beraudo, J. G. Milhano, and U. A. Wiedemann (in preparation).

[12] B. R. Webber, Nucl. Phys. B 238, 492 (1984).

[13] K. J. Eskola, H. Honkanen, C. A. Salgado, and U. A. Wiedemann, Nucl. Phys. A 747, 511 (2005).

[14] S. Sapeta and U. A. Wiedemann, Eur. Phys. J. C 55, 293 (2008).

[15] K. Zapp, G. Ingelman, J. Rathsman, J. Stachel, and U. A. Wiedemann, Eur. Phys. J. C 60, 617 (2009). 